\title{
Comparing methods of ileostomy closure constructed in colorectal surgery in Turkey
}

\author{
Bulent Dinc ${ }^{1}$, Nurettin $\mathrm{Ay}^{2}$, Huseyin Ciyiltepe ${ }^{3}$ \\ ${ }^{1}$ Department of Surgery, Ataturk State Hospital, Antalya, Turkey \\ 2Department of Surgery, Diyarbakir Training and Research Hospital, Diyarbakir, Turkey \\ ${ }^{3}$ Department of Surgery, Faculty of Medicine, Akdeniz University, Antalya, Turkey
}

Prz Gastroenterol 2014; 9 (5): 291-296

DOI: $10.5114 / p g .2014 .46165$

Key words: loop ileostomy, surgical closure techniques, anastomosis.

Address for correspondence: Bulent Dinc MD, Ataturk State Hospital, Department of General Surgery, Gulluk Street, 07040 Antalya, Turkey, phone: +90 24234545 50, fax: +90 24233433 73, e-mail: bulent1999@yahoo.com

\begin{abstract}
Introduction: Stoma construction is a life saver method for emergent and elective operations in colorectal surgery. However, they are associated with high rates of morbidity and mortality.

Aim: To compare the operative findings, early postoperative complications, and costs of stapled and hand-sewn closures in loop ileostomies that are constructed in emergent and elective colorectal surgery.

Material and methods: The data of 68 patients requiring loop ileostomies during colorectal surgery were retrospectively evaluated. SPSS (version 20) was used for data analysis.

Results: The study group consisted of 44 men and 24 women with a mean age of 55.5 years. The ileostomy closures were performed with hand-sewn method in 36 patients (group 1) and stapled method in 32 patients (group 2). The mean operation time was $75.4 \mathrm{~min}$ in group 1 and $46.7 \mathrm{~min}$ in group 2 ( $p<0.001)$. Early postoperative complications were wound infection $(8.8 \%)$, small bowel obstruction (6.06\%), and anastomotic leakage (2.9\%). Total costs, flatulence and faeces outlet time, oral feeding starting time, time of hospital stay, and early postoperative complications were lower in the stapled group.

Conclusions: Morbidity and mortality rates of stoma construction and its closure are still considerable. Lower anastomotic leakage rate, complication rate, and costs and shorter operative times in the stapled group make this method preferable.
\end{abstract}

\section{Introduction}

Stoma operations are life-saving operations performed either under urgent or elective circumstances $[1,2]$. Today, along with progress in anastomosis techniques, more radical surgical procedures can be performed, and stomas are widely used in these procedures in order to prevent anastomosis leakage. Especially after surgical procedures with low anastomosis safety, loop ileostomy performance for faecal diversion is reducing morbidity and mortality $[3,4]$.

Stoma procedures may cause complications during the postoperative period. In literature, these complications are reported to be between $10 \%$ and $50 \%$ [1]. It is thought that these complications can be reduced by correct stoma opening indications and by use of better surgical techniques during closure [5].

\begin{abstract}
Aim
The aim of this study is to compare the anastomosis techniques for loop ileostomy closure, performed with stapler or manually, that were performed during urgent or elective colorectal surgery, by means of operative findings, early complications, and cost benefit analysis, and to explore the most suitable and reliable technique.
\end{abstract}

\section{Material and methods}

Loop ileostomy closure operations were retrospectively studied of 68 patients who underwent urgent or elective loop ileostomy during colorectal surgery in Akdeniz University Medical School, Department of General Surgery between January 2008 and December 2012. Randomly selected patients that were manually closed 
were called group 1 and patients that were closed by using stapler were called group 2.

By using the hospital records, the following parameters were compared between the groups: patient age, gender, comorbidity problems, preoperative albumin and haemoglobin levels, reasons for stoma procedure, duration between two operations, operative findings (operative technique, duration of operation), postoperative status (flatulence and faeces output and duration of oral feeding, duration of hospital stay), complications (wound infections, intestinal obstructions, complications ligated to anastomosis), and cost benefit analysis.

Informed approval of surgical intervention was obtained from each patient preoperatively. Oral feeding was stopped the night before the operation and no mechanical intestinal cleaning was performed. All patients received second-generation cephalosporin prophylaxis (Cefazolin $1 \mathrm{~g}$ ) during induction of anaesthesia. In patients with complications, Ceftriaxone $(2 \mathrm{~g} /$ day IV - twice a day) was added to medication. All of the operations were performed by experienced surgeons.

Anastomosis was carried out using two different techniques. In the first group, side-to-side anastomosis by Connell suture technique following partial resection was performed. During the procedure, 3/0 - 4/0 Polyglactine (Vicrly) for full layer sutures and 3/0 silk material for serosa sutures were used. In the second group, anastomosis was made side-to-side by means of a stapler. During anastomosis, two GIA (Gastrointestinal Anastomosis) 80/3.8 mm linear cutter staplers were used. With the first, anastomosis between two intestinal loops were performed and with the second, open intestinal ends were sealed.

Following the day of flatulence, oral feeding with liquids was started. After oral intake, IV fluid support was stopped and the patients were discharged. The length of stay in hospital was described as the duration between the day of operation and the day of discharge.

Wound infection and early-phase intestinal obstruction in the first postoperative 30 days was described as a minor complication.

In the patients with intestinal content fistulasing from the incision area or with abdominal sepsis findings supporting clinical anastomosis leakage, radiological findings affirming intestinal content in the operation area or intra-abdominal abscess were defined as "anastomosis leakage". Patients with anastomosis leakage were reoperated immediately.

Preoperative and postoperative morbidities and mortalities in the first 30 days were taken into account.

Costs concerning operative and postoperative follow-ups were obtained from hospital records.

\section{Statistical analysis}

Results of surgical techniques for patients in whom ileostomies were closed in two different techniques were compared. Data were analysed by means of SPSS (v. 2.0 - SPSS Inc., Chicago, USA). For defining samples, definitive sampling statistics such as frequency modulation, means, and standard deviation were used. Difference between the two groups were explored using Student $t$ test, two pair test, variant analysis, Mann Whitney $U$ test, Wilcoxon test, and Kruskall Wallis test. Relations between continuous variables were analysed by Spearman correlation coefficient. Categorical variables were explored by means of the $\chi^{2}$ test. For determining differences between groups, a $95 \%$ significance level was used.

\section{Results}

Of the 68 patients undergoing operation for ileostomy closure, 44 were male (64.7\%) and 24 were female (35.3\%) and mean age was 55.5 years (range: 26-85 years). Comparison for age, gender, comorbidity problems, and illnesses for stoma procedures between groups are given in Table I.

No statistical differences between groups for demographic variables were seen. In Table I, it can seen that the most frequent indication for stoma procedures for both groups was low anterior resection (LAR) operation for rectal tumours (52/68, 76.5\%).

Disregarding the groups, 18 patients (26.5\%) had anaemia (haemoglobin levels under $11 \mathrm{~g} / \mathrm{dl}$ ), 7 patients (10\%) had hypoalbuminaemia (albumin levels under $3 \mathrm{~g} / \mathrm{dl})$.

In group 1, 9 patients (25\%) had anaemia and 4 patients $(11.1 \%)$ had hypoalbuminaemia, and in group 2, 9 patients (28.1\%) had anaemia and 3 patients (9.3\%) had hypoalbuminaemia. For preoperative haemoglobin and albumin levels of the patients, there were no significant differences between the groups $(p>0.05)$.

The mean time between primary surgery and ileostomy procedure was calculated to be 168 days (34475 days). This duration was 182 days in group 1 and 153 days in group 2, and no significant difference was seen $(p>0.05)$.

For the rest of the patients, mean operation time was 61.9 min (40-110 min), mean postoperative flatulence or faeces outlet time was 2.96 days (1-8 days), mean oral feeding starting time was 3.88 days (2-9 days), and mean time of hospital stay was 5.6 days (3-36 days). A comparison between the groups is shown in Table II.

As postoperative complications, wound infections, early phase intestinal obstruction and anastomosis leakages were evaluated. In 6 of 68 patients (8.8\%) 
Table I. Demographic comparison of group 1 and group 2

\begin{tabular}{|c|c|c|}
\hline Parameters & Group 1, $n=36$ & Group 2, $n=32$ \\
\hline Age, mean [years] & 55.9 & 55.2 \\
\hline \multicolumn{3}{|l|}{ Gender, $n(\%)$ : } \\
\hline Female & $13(36.2)$ & $11(34.3)$ \\
\hline Male & $23(63.8)$ & $21(65.6)$ \\
\hline \multicolumn{3}{|l|}{ Comorbid diseases, $n$ : } \\
\hline Hypertension & 7 & 7 \\
\hline Diabetes mellitus & 4 & 5 \\
\hline Coronary artery disease & 4 & 3 \\
\hline Chronic obstructive pulmonary disease & 1 & 2 \\
\hline Others (atrial fibrillation, pulmonary embolism, chronic renal failure) & 3 & 3 \\
\hline \multicolumn{3}{|l|}{ Causes of stoma, $n$ : } \\
\hline Rectal tumour & 28 & 24 \\
\hline Family adenomatous polyposis (FAP) & 5 & 3 \\
\hline Ulcerative colitis & 1 & 4 \\
\hline Aganglionic megacolon & 1 & 0 \\
\hline Rectal injuries & 1 & 0 \\
\hline Colocutaneous fistula & 0 & 1 \\
\hline
\end{tabular}

Table II. Comparison of duration of operation, duration of gas-faeces output, time to oral feeding, and length of hospital stay

\begin{tabular}{lccc} 
Parameters & Group 1 & Group 2 & \multirow{2}{*}{ Value of $p$} \\
\cline { 2 - 3 } & \multicolumn{2}{c}{ Mean (range) } & $<0.001$ \\
\hline Operation time [h] & $75.41(40-110)$ & $46.71(40-80)$ & 0.03 \\
\hline Flatulence or faeces outlet time [days] & $3.55(1-8)$ & $2.37(1-5)$ & 0.02 \\
\hline Oral feeding starting time [days] & $4.50(2-9)$ & $3.26(2-5)$ & 0.01
\end{tabular}

wound infections were encountered. In patients with wound infections, incision sutures were removed and daily wound dressings were applied and IV antibiotherapy was continued. All the patients were discharged without any further surgical intervention. In 4 patients (6.06\%), intestinal obstruction evolved in the early postoperative phase. These patients were followed up with conservatory approaches and were discharged without complication. Only 2 of 68 patients $(2.9 \%)$ were diagnosed with anastomosis leakage. Both of the patients were in group 1 and were diagnosed as developing anastomosis leakage on the third postoperative day. They were reoperated and ileostomy was performed again. After the second surgical procedure, the patients were followed up in the intensive care unit (ICU), and after discharge wide spectrum antibiotherapy was continued. Both of the patients' length of hospital stay increased, but they were discharged from hospital without any additional medical or surgical complications. Total rate of complications was $17.6 \%$ with wound infections in first place (6 patients, $8.8 \%$ ). A comparison between groups for complications can be seen on Table III. No surgical mortality was seen in the patients.

When total cost analysis is evaluated, it can be seen that costs are higher for group $1(\$ 1323)$ than for group 2 (\$831). The difference is statistically significant $(p=0.018)$. What makes the difference between the groups is the anastomosis leakages and long hospitalisation time in group 1.

\section{Discussion}

Surgeons tend to perform protective loop ileostomy in order to prevent pelvic sepsis due to anastomosis 
Table III. Distribution of total complications according to groups

\begin{tabular}{|c|c|c|c|}
\hline Complications & Group $1(n=36)$ & Group $2(n=32)$ & Value of $p$ \\
\hline \multicolumn{4}{|l|}{ Minor: } \\
\hline Wound infection & $4(11.1 \%)$ & $2(6.2 \%)$ & 0.67 \\
\hline Small bowel obstruction & $2(5.5 \%)$ & $2(6.2 \%)$ & 0.79 \\
\hline \multicolumn{4}{|l|}{ Major: } \\
\hline Anastomosis leakage & $2^{\star}(5.5 \%)$ & 0 & $<0.001$ \\
\hline Total complications & $8(22.2 \%)$ & $4(12.5 \%)$ & 0.29 \\
\hline
\end{tabular}

*Surgical requirement

leakage in patients with distal colonic anastomosis [6]. The prevalence of clinically or radiologically diagnosed anastomosis leakage following distal colon resections is nearly $17 \%$ and it is higher in sphincter-protecting surgical operations [7]. Anastomosis leakages may eliminate the chance of lower cited anastomosis, and patients may be obliged to live with permanent stomas. Loop ileostomy does not prevent anastomosis leakages but in the case of leakage, it reduces the severity of pelvic sepsis [8].

Times for ostomy closure differ in literature. For example, according to a study on 1504 patients of restorative proctocolectomy by Wong et al. [9], the mean time was determined to be 98 days (9 days -38.3 months). When the results of the meta-analysis by Chow et al. [10] are studied, it can be seen that the time between primary surgery and ostomy closure is shorter in restorative proctocolectomy patients that in rectum cancer patients.

In a meta-analysis by Hindenburg and Rosenburg [11] it was declared that closure of ileostomy in the early phase (< 14 days), normal phase (14-100 days), or late phase ( $>100$ days) does not have a significant effect on postoperative mortality and morbidity. But early closure of the ileostomy is found to decrease morbidity related to stoma.

We calculated the mean time between primary surgery and operation for ileostomy closure as 168 days (34-475 days) in our study. The mean time was 178 days in patients who underwent LAR operation and 139 days for other operations. The reason why there is no difference in the mean times might be the excess of patients requiring operation, and therefore the delayed schedule.

In a study by Chow et al. [10], duration of operation is stated to have a mean of $63.5 \mathrm{~min}$. Studies by Hasegawa et al. [12], Leung et al. [13], and Shelygin et al. [14] declare that operations using a stapler take less time than ones made manually. In our study, mean duration of operation is seen to be $61.9 \mathrm{~min}$. Consistent with literature, operations with use of a stapler statistically significantly took less time.
In a study by Chow et al. [10], it was noted that intestinal activation started after a mean of 2.1 days and defecation was seen after a mean of 2.2 days. In a study published by Balık et al. [15], it was stated that the duration of operations performed manually or with stapler were similar, although patients operated on with a stapler had a shorter period for flatulence and faeces outlet and they were discharged sooner. Peacock et al. [7] and Kalady et al. [16] reported that it is safe to discharge patients undergoing ileostomy closure operation after $23 \mathrm{~h}$ of observation following oral feeding. In our study, the durations are similar with literature. The durations are shorter in group 2. The reason why the patients were discharged later despite the fact that oral feeding began earlier might be related to the fact that most of the operated patients came from distant locations, and the consequent difficulty in admission to hospital, and for this reason, the tendency of doctors to be sure of oral feeding of patients before discharge.

There are many studies comparing the morbidities of the two techniques. It was seen that morbidity rates of studies were $21.5 \%$ for Luglio et al. [3], 19.4\% for Balık et al. [15], 11.4\% for Wong et al. [9], and 17.3\% for Chow et al. [10], but no differences were seen between groups in any of the studies. The total morbidity rate of $17.6 \%$ in our study is consistent with literature.

One of the most frequent complications of ileostomy closure procedure is wound infection. Despite different rates, it is seen in an average of $18.3 \%$ of cases. Rate of wound infections was $5 \%$ in Chow's study [10] and $9.2 \%$ in Hasegawa's study [12], and no differences between groups were seen in either study. In our study, wound infection incidence was $8.8 \%$, and it was higher in group 1, but no statistical difference was seen between the two groups. Despite statistical indifference, we conclude that the higher wound infection incidence in the manual anastomosis group is related to the fact that the wound site may not be adequately protected from intestinal content. Manual closure's more difficult technique and longer operation duration seem to be factors effecting wound infections. 
Intestinal obstructions following ileostomy closure procedures are reported to be between $0 \%$ and $15 \%$ in literature. There are investigators stating that intestinal obstructions are more rare in operations performed with a stapler as these operations require larger diameter anastomosis [9]. In a meta-analysis by Chow et al. [10], intestinal obstruction incidence was stated to be $7.2 \%$. In our study, $6.06 \%$ of patients developed intestinal obstruction and the patients were treated by conservative methods. Relaparotomy was not needed, but length of hospital stay increased in these patients. Intestinal obstruction was thought to be related to primary surgery.

The most important complication of ileostomy closure is anastomosis leakage. Its approximate frequency is between 0 and $10 \%$. It is a serious complication that may cause intra-abdominal abscess, enteric fistulas, or septic shock. Usually it is treated with surgical methods. In our study, the incidence was determined to be a little higher (2.9\%). As anastomosis leakages were seen in the manually operated group, anastomosis with a stapler seems to be safer. The technical difficulty of manual anastomosis might be a reason for this increased safety. Moreover, anastomosis may be lower risk as side-to-side anastomosis technique is used in manual anastomosis. Larger patient groups are needed to evaluate this.

Operative mortality in ileostomy closure is low. In literature, mortality after ileostomy closure is said to be between $1 \%$ and $6.4 \%$. The highest rate in literature is declared by Zelkowicz et al. [17]. In this study, three patients died of aspiration pneumonia and 1 patient of septic shock following postoperative ischemic colitis.

In a study by Mansfield et al. [18], mortality is stated to be $3.3 \%$. In this study, mortalities were due to mesentery infarct, postoperative pulmonary infection, postoperative duodenal ulcer perforation, and ileo-ileal anastomosis leakage. Mortality rates were $0.4 \%$ in Chow's study [10] and $0.06 \%$ in Wong's study [9]. There were no operative mortalities in our study.

There are few studies on total hospital costs for ileostomy closure procedures in literature. In a study by Horisberger et al. [19] cost related to duration of operation was lower in the stapler group because the duration of operation is shorter with a stapler. Although a stapler is an expensive tool, it was shown in this study that shortening the duration of operation reduces the total hospital cost. In our study, total cost with manual technique was significantly higher than total cost with a stapler. We relate this higher cost with manual technique to the onset of major complications like anastomosis leakage in two patients. It should be kept in mind that both the performance and closure of the ileostomy have high morbidity. Although no statistical differences were achieved, lower anastomosis leakage risk with a stapler compared to manual technique gives the impression that stapler usage is safer. As the risk of complications with manual anastomosis is higher, total costs increase in this group. Moreover, stapler usage reduces the duration of operation and therefore lowers total hospital costs. The length of anaesthesia shortens and indirectly helps postoperative healing. We think that prospective randomised trials containing more patients would be helpful in comparing the two groups.

\section{Conclusions}

Morbidity and mortality rates of stoma construction and its closure are still considerable. The lower anastomotic leakage rates, lower complication rates, lower costs, and shorter operative times in the stapled group will make this method preferable.

\section{References}

1. Sharma A, Deeb AP, Rickles AS, et al. Closure of defunctioning loop ileostomy is associated with considerable morbidity. Colorectal Dis 2013; 15: 458-62.

2. Caricato M, Ausania F, Ripetti V, et al. Retrospective analysis of long-term defunctioning stoma complications after colorectal surgery. Colorectal Dis 2007; 9: 559-61.

3. Luglio G, Pendlimari R, Holubar DS, et al. Loop ileostomy reversal after colon and rectal surgery, a single institutional 5-year experience in 944 patients. Arch Surg 2011; 146: 1191-6.

4. Courtier R, Parés D, Silva CA, et al. Clinical results of loop ileostomy closures in rectal cancer surgical patients. Effect of chemotherapy in the waiting period. Cir Esp 2010; 88: 308-13.

5. Bafford AC, Irani JL. Management and complications of stomas. Surg Clin North Am 2013; 93: 145-66.

6. Perez RO, Habr-Gama A, Seid VE, et al. Loop ileostomy morbidity: timing of closure matters. Dis Colon Rectum 2006; 49: 1539-45.

7. Peacock O, Law Cl, Collins PW, et al. Closure of loop ileostomy: potentially a day case procedure? Tech Coloproctol 2011; 15 : 431-7.

8. Mengual-Ballester M, García-Marín JA, Pellicer-Franco E, et al. Protective ileostomy: complications and mortality associated with its closure. Rev Esp Enferm Dig 2012; 104: 350-4.

9. Wong KS, Remzi FH, Görgün E, et al. Loop ileostomy closure after restorative proctocolectomy: outcome in 1504 patients. Dis Colon Rectum 2005; 48: 243-50.

10. Chow A, Tilney HS, Paraskeva P, et al. The morbiditi surrounding reversal of defunctioning ileostomies: a systematic review of 48 studies including 6,107 cases. Int J Colorectal Dis 2009; 24: 711-23.

11. Hindenburg T, Rosenburg J. Closing a temporary ileostomy within two weeks. Dan Med Bul 2010; 57: A4157.

12. Hasegawa H, Radley S, Morton DG. Stapled versus sutured closure of loop ileostomy: randomized controlled trial. Ann Surg 2000; 231: 202-4. 
13. Leung TT, MacLean AR, Buie WD, et al. Comparison of stapled versus handsewn loop ileostomy closure: a meta-analysis. J Gastrointest Surg 2008; 12: 939-44.

14. Shelygin YA, Chernysov SV, Rybakov EG. Stapled ileostomy closure results in reduction of postoperative morbidity. Tech Coloproctol 2010; 14: 19-23.

15. Balık E, Eren T, Buğra D, et al. Revisiting stapled and handsewn loop ilestomy closures: a large retrospective series. Clinics 2011; 66: 1935-41.

16. Kalady MF, Fields RC, Klein S, et al. Loop ileostomy closure at an ambulatory surgery facility. Dis Colon Rec 2003; 46: 486-90.

17. Zelkowicz BF, Cazador AC, Coll RF, et al. Morbidity and mortality associated with diverting ileostomy closures in rectal cancer surgery. Cir Esp 2008; 1: 16-9.

18. Mansfield SD, Jensen C, Phair AS, et al. Complications of loop ileostomy closure: a retrospective cohort analysis of $123 \mathrm{pa}$ tients. World J Surg 2008; 32: 2101-6.

19. Horisberger K, Beldi G, Candinas D. Loop ileostomy closure: comparison of cost effectiveness between suture and stapler. World J Surg 2010; 34: 2867-71.

Received: 7.12.2013

Accepted: 2.01.2014 